\title{
Historias de familia. El marco ampliado de las historias de vida
}

\author{
Family Stories. An Extended Framework of Life Stories \\ Histórias de família. O marco ampliado das histórias de vida \\ Cristina Álvarez Vargas ${ }^{1}$ \\ Juan Carlos Amador Baquiro²
}

\section{Resumen}

El artículo explora qué es y cómo fue usado el método historias de familia (Bertaux, 1995; Miller, 2007) en la investigación doctoral "Experiencias de crianza en contextos de conflicto armado en Colombia". El texto expone cómo se ubica este método en los debates contemporáneos sobre investigación cualitativa y su articulación epistemológico-metodológica. Luego muestra la relación de este método con la tradición biográfico-narrativa de la investigación social. Después, explica cómo surgió el método a partir de lo que Bertaux (1995) llama historias de familia desde la genealogía social comparada y comentada (GSCC). Más adelante, describe los criterios conceptuales y procedimentales de las historias de familia a partir del modelo sugerido por Miller (2007). Por último, se incluyen algunos ejemplos de la investigación doctoral que ilustran el uso del método.

\section{Palabras clave}

historias de familia; investigación cualitativa; métodos biográfico-narrativos; narrativa; conflicto armado

\section{Abstract}

This paper inquires about what the family story approach (Bertaux, 1995; Miller, 2007) is and how it was used in a doctoral research entitled "Upbringing experiences in armed conflict contexts in Colombia". The text describes the point of view of this approach in contemporary discussions on qualitative research and its epistemological-methodological articulation. Then, it shows the relationship between this method and the biographical-narrative tradition of social research. After that, it explains how the approach emerged from what Bertaux (1995) calls family stories in his Commented and Compared Social Genealogies. Later, it describes the conceptual and procedural criteria of family stories from Miller's approach (2007). Finally, it includes some examples from the doctoral research illustrating how the method is used.

\section{Keywords}

family stories; qualitative research; biographical-narrative approaches; narrative; armed conflict

\section{Resumo}

0 artigo explora o que é e como foi usado o método histórias de família (Bertaux, 1995; Miller, 2007) na pesquisa doutoral “Experiências de criação em contextos de conflito armado na Colômbia". 0 texto expõe como se localiza esse método nos debates contemporâneos sobre pesquisa qualitativa e sua articulação epistemológico-metodológica. Em seguida, evidencia a relação desse método com a tradição biográfico-narrativa da pesquisa social. Depois, explica como surgiu o método a partir do que Bertauz (1995) chama de histórias de família desde a genealogia social comparada e comentada (GSCC). Logo após descreve os critérios conceptuais e procedimentais das histórias de família a partir do modelo sugerido por Miller (2007). Finalmente, inclui alguns exemplos da pesquisa que ilustram o uso do método.

\section{Palavras chave}

histórias de família; pesquisa qualitativa; métodos biográfico-narrativos; narrativa; conflito armado

Artículo recibido el 18 de mayo del 2016 y aprobado el 21 de diciembre del 2016

1 Candidata a Doctora en Ciencias Sociales, Niñez y Juventud de la Fundación Cinde y la Universidad de Manizales, Colombia. Docente de Pedagogía Infantil de la Universidad Distrital Francisco José de Caldas y de la Maestría en Educación y Desarrollo Humano del convenio Fundación Cinde y la Universidad de Manizales, Colombia. Correo electrónico: cristyalvarez77@gmail.com

2 Director de la línea de investigación en "Familias e infancias en la cultura" del Doctorado en Ciencias Sociales, Niñez y Juventud de la Fundación Cinde y la Universidad de Manizales, Colombia. Correo electrónico: juanamador@udistrital.edu.co 


\section{Introducción}

Durante las últimas tres décadas, los estudios sobre familias en las ciencias sociales han provocado un giro analítico-interpretativo de gran relevancia en relación con objetos de estudio convencionales, especialmente situados en la psicología y la medicina, los cuales, históricamente, han abordado la constitución, la estructura o las dinámicas de las familias a partir de taxonomías sociales y parentales, patologías e intervenciones. Tras las nuevas realidades que configuran las familias contemporáneas, como la migración, la precarización laboral, las violencias asociadas con la desigualdad y la exclusión, y el debilitamiento de lo societal como expresión de la reducción de espacios democráticos, se han ido desnaturalizando ciertos procesos asociados con la maternidad y la paternidad, el matrimonio, la crianza y los procesos de socialización, entre otros aspectos adscritos al modo de funcionamiento de la familia moderna (Burin y Meler, 2003).

Particularmente los estudios culturales, las perspectivas feministas, los estudios decoloniales y algunos enfoques críticos, ubicados en disciplinas como la antropología, la sociología, la historia, la psicología y el trabajo social, han mostrado mediante investigaciones cómo se están transformando las familias a partir de tanto nuevas formas de vínculo y parentesco, otros modos de maternidad y crianza, como formas emergentes de socialización y educación de los hijos e hijas tras la presencia de nuevas masculinidades y feminidades. Sin embargo, más allá de los debates teóricos que orientan las investigaciones contemporáneas sobre familias, los cuales no serán desarrollados acá, es importante reflexionar sobre los métodos de investigación que podrían aportar criterios y procedimientos útiles para continuar en este proceso de deconstrucción de conceptos, discursos y textos sobre las familias contemporáneas.

En esta dirección, el presente artículo expone los principales aspectos que constituyen el método denominado "historias de familia" (Miller, 2000, 2007). Como se evidenciará más adelante, se trata de un método que tiene su origen en la tradición cualitativa y biográfico-narrativa de los estudios en ciencias sociales, lenguaje, filosofía y educación, y que emplea herramientas interpretativas asociadas con la sociogénesis y las genealogías sociales abordadas por ciertas ramas de la sociología para comprender las permanencias y cambios de estas agrupaciones sociales. Además de exponer los fundamentos y procedimientos del método, se presentarán algunos análisis y problemas que surgieron como parte de su aplicación en la investigación doctoral titulada "Experiencias de crianza en contextos de conflicto armado en Colombia" (Álvarez, 2015) ${ }^{3}$.

Otro elemento importante a tener en cuenta es que el método proporciona criterios para abordar problemas de investigación orientados hacia la construcción de memorias a partir de los vínculos o tensiones entre las biografías personales, las historias colectivas de la familia y la temporalidad social más amplia. Por esta razón, el método fue útil para la investigación en mención, pues no solo se trataba de dar cuenta de las historias de familia en general, sino de crear puentes para develar cómo los acontecimientos vividos por un sujeto en la experiencia de la guerra guardan continuidades, pero también evidencian rupturas, frente a lo ocurrido con otros integrantes de su familia pertenecientes a otra generación que también tuvieron que enfrentar el conflicto armado. En suma, además de posibilitar la lectura de lo intergeneracional, el método contribuye a develar memorias desde lugares no hegemónicos y a problematizar las temporalidades de los subalternos con la temporalidad hegemónica (parental, institucional, nacional) (Spivack, 2003).

A continuación, se expondrá cómo se ubica dicho método en los debates contemporáneos sobre investigación cualitativa y su articulación epistemológico-metodológica. Luego se mostrará la relación de este con la tradición biográfico-narrativa de la investigación social. Después se explicará cómo

3 La investigación mencionada es el marco en el que se desarrolla esta metodología y se lleva a cabo actualmente para optar por el título de Doctora en Ciencias Sociales, Niñez y Juventud. 
surgió el método a partir de lo que Bertaux (1999) llama historias de familia desde la genealogía social comparada y comentada (GSCC). Más adelante, se describirán los criterios conceptuales y procedimentales de las historias de familia a partir del modelo sugerido por Miller (2007). Por último, se incluirán algunos ejemplos de la investigación doctoral que ilustran el uso del método.

\section{La investigación cualitativa: articulación epistemológica y metodológica}

Algunos debates reconocidos en el campo de la investigación cualitativa (Kauffman, 2005) coinciden en que no solo se trata de una estrategia para recolectar, sistematizar y analizar datos, sino especialmente de un enfoque que busca dar cuenta del sujeto conocido (Vasilachis, 2006). En esta dirección, es necesario partir de cuatro presupuestos que plantean, desde lo cualitativo, una imbricación estratégica entre epistemología y metodología. Basados en Vasilachis (2006), Coffey y Atkinson (2003) y Morse (2003), estos presupuestos pueden sintetizarse así: resistencia a la naturalización del mundo social; revaloración del concepto de mundo de la vida; tránsito de la observación a la comprensión; e implementación de una doble hermenéutica.

El primer presupuesto se origina en la noción de realidad social que, tempranamente, distinguieron Dilthey y Mannheim, entre otros. En primer lugar, Dilthey (citado en Berger y Luckman, 2005), como precursor del historicismo, propuso los términos "determinación situacional" y "asiento en la vida", con lo que intentó ofrecer una base explicativa acerca de la ubicación social de la realidad y del pensamiento. Por su parte, Mannheim (citado en Berger y Luckman, 2005) acuñó el término "relacionismo", en contraposición al relativismo, para dar cuenta de los componentes que delimitan el conocimiento social, esto es, un tipo de conocimiento que requiere del análisis sistemático para comprender tanto las bases de su diversidad social y los intersticios de los grupos sociales que indican su variabilidad a pesar de estar organizados por clases, como la utopía, comprendida como una fuerza dinámica capaz de transformar la propia realidad de la que subyace.

El segundo presupuesto también resulta potente para la producción de conocimiento social. El mundo de la vida - lebenswelt - , tal como lo concibió Edmund Husserl (citado en Berger y Luckman, 2005), remite a la existencia de condiciones temporales, espaciales, éticas, políticas, estéticas y culturales en las que tiene lugar la acción humana y sus respectivos posicionamientos. El mundo de la vida en el terreno de la fenomenología plantea la necesidad de conocer los aspectos constitutivos de los fenómenos, más allá de la representación y su intento por hacer presente lo ausente. Por consiguiente, el mundo de la vida es el espacio-tiempo en el que se dan los procesos de entendimiento, conducentes a otorgar los recursos necesarios para favorecer las prácticas y las interacciones que tienen lugar a través de la intersubjetividad y las estructuras de la vida. Según Vasilachis (2006), este es un escenario que ofrece a los actores patrones y opciones para transitar por las experiencias mediante un constante trasegar por horizontes que dan cabida a la existencia con otros, en marcos sociales y culturales contingentes y dinámicos.

En relación con el tercero y último presupuesto, aquellos que aluden al tránsito de la observación a la comprensión y a la doble hermenéutica, es claro que los debates sobre el conocimiento y la interpretación en las ciencias sociales, por ejemplo en torno a asuntos como la relación entre cocimiento e interés (Habermas, 2007), la investigación de segundo orden (Ibañez, 1990), la interculturalidad crítica (Walsh, 2012) y la hermenéutica diatópica (Santos, 2009), evidencian que el intérprete de la realidad no es sólo un traductor o un avezado explorador que se atreve a formular explicaciones. El investigador no puede dedicarse exclusivamente a producir significados acerca de lo que observa sino a hacer explícitos los universos de significados a través de los cuales los sujetos y grupos tramitan su propia interpretación, en el marco de una disposición permanente de estos a participar en la empresa del mundo social. 


\section{Los estudios biográfico-narrativos}

En la tradición cualitativa de las ciencias sociales, desde la década de los setenta fue evidente la tendencia a investigar fenómenos asociados con la interacción social, las identidades culturales y las subjetividades, situación que fue propiciando el abandono de relatos y métodos propios de las ciencias sociales modernas (nomotéticas e ideográficas). Esto hizo que los estudios sociales se orientaran hacia la pluralidad y la polifonía de voces, y hacia la multiplicidad de experiencias y saberes situados en la periferia y en las fronteras más que en el centro y los lugares hegemónicos. En este contexto, surgieron los enfoques y métodos biográficos en las ciencias sociales, comprendidos como una perspectiva que "enfatiza la posición y participación de los individuos dentro de una estructura social existente" (Miller, 2000, p. 2). Estas investigaciones inicialmente fueron desarrolladas a través del trabajo de profesionales de diversas disciplinas, entre ellas la lingüística, la antropología, la sociología, la psicología y la ciencia política.

La tradición biográfica de la investigación cualitativa (Creswell, 1998), en sus acepciones relato de vida, historia de vida y autobiografía, coinciden en que no solo se trata de reconstruir los hechos significativos de las personas sino de comprender cómo estos adquieren alguna relación con el contexto histórico y la posición del sujeto en la estructura social. Asimismo, implica la reflexividad de sus protagonistas a partir de ejercicios de rememoración que tienden a relacionar el presente con el pasado (Miller, 2000), y a develar la multiplicidad de redes de relaciones que los sujetos atraviesan y adscriben en la vida cotidiana (Ferraroti, 1988).

La introducción de la narrativa en la investigación social hace parte de lo que Denzin (citado en Coffey y Atkinson, 2003) ha llamado giro interpretativo. La narrativa, comprendida como el interés permanente de las personas por organizar mediante alguna secuencia acontecimientos de su vida social o íntima con el fin de otorgar sentido tanto al propio narrador como a un público potencial, contiene importantes alternativas para producir la doble hermenéutica planteada. Esto es así al tener en cuenta que en la narrativa las personas se posicionan de ciertas maneras tanto para contar historias, coordinar sucesos alrededor de tiempos, espacios y personajes, como para dilucidar interpretaciones y reacciones a los hechos que emergen en estructuras con diversos significados, formatos, contenidos y recursos.

Basados en la propuesta de Coffey y Atkinson (2003) sobre la investigación narrativa, se puede plantear una sencilla clasificación de niveles como alternativa para dilucidar el método historias de familia. El primero obedece al nivel estructural de la narrativa, es decir, a una interpretación organizada alrededor de aquello que se cuenta, a través de preguntas. Si se parte de que en una narrativa existen tres momentos -inicio, nudo y desenlace-, entonces la introducción de algunas preguntas orientadoras puede contribuir a un reconocimiento riguroso del contenido en su conjunto así: "sobre el resumen (¿de qué trata?), sobre la orientación (¿qué?, ¿quién?, ¿cuándo?, ¿cómo?), sobre la complicación (¿entonces qué sucedió?), sobre la evaluación (¿y entonces qué?), sobre el resultado (¿finalmente qué pasó?), y sobre la coda (¿cómo termina la narrativa?)" (Coffey y Atkinson, 2003, p. 69).

El segundo nivel está especialmente ligado a las funciones espacio-temporales de las narrativas. Se trata del conjunto de acciones sociales implícitas en los textos, lo que supone apartarse de la estructura lingüística de la narrativa y acercarse más a los universos socioculturales que configuran los contextos en los que esta tiene lugar. Generalmente las narrativas individuales están situadas dentro de interacciones particulares y en el marco de discursos y prácticas institucionales, culturales o sociales específicos. Para tal efecto, esta mirada a las narrativas, más contextual que estructural, puede apelar a relatos de éxitos morales, a narrativas al estilo crónica, y a etnopoéticas (Coffey y Atkinson, 2003). Aunque esta delimitación puede resultar arbitraria, es bastante útil dado que contribuye a develar el entrecruzamiento permanente entre las funciones de identidad con las de rememoración, 
de evaluación moral, de interpelación, de acomodación en estructuras organizacionales, de compromiso social y de entretenimiento presentes en la narrativa.

En el tratamiento de los relatos de éxitos morales el investigador debe analizar los múltiples recursos utilizados por el narrador para mostrar la magnitud de sus éxitos y la superación de un conjunto de obstáculos que le han permitido llegar a determinadas metas. Desde luego, es algo que tiende a ocurrir bajo la presencia, casi obligada, de una moraleja que le facilita proceder a la inclusión de cierres moralizantes. Por su parte, la narrativa como crónica es una estructura ordenada de sucesos en la que el protagonista se permite hacer profundizaciones $y$ matizaciones, atendiendo a valoraciones y juicios. En algunas ocasiones la crónica puede estar intervenida por dimensiones estructurales y arreglos sociales que se evidencian en la narrativa, pero que escapan del control del protagonista. En suma, la idea de crónica es planteada por Coffey y Atkinson (2003) como un nivel narrativo más complejo, dado que exige transitar tanto por textualidades e intertextualidades, como por narrativas colectivas y autobiográficas.

Por último, la etnopoética alude a la función sociocultural de la narrativa como medio para construir y compartir valores culturales, significados y experiencias personales. Parte de la identificación de expresiones y palabras que adquieren fuerzas autónomas en la vida social y cultural a través de los usos y apropiaciones efectuados en los contextos de la acción. El mayor reto para el investigador es comprender la lógica en la que son empleadas metáforas, hipérboles y analogías en la vida de un grupo social o de una institución, en los momentos de producción y de ocio, o simplemente en torno de aquello que autores como Lakoff y Johnson (1980) denominan metáforas en la vida cotidiana. Dentro de esta tipología, lo sociocultural, lo lingüístico y lo literario se funden alrededor de una estilística, cuya narración también da cuenta de los acontecimientos y del devenir de los sujetos y los grupos que, en el día a día, se ven enfrentados a problemas, deseos y conflictos.

\section{Las historias de familia desde la genealogía social comparada y comentada}

Daniel Bertaux (1999), otro importante referente en la investigación biográfico-narrativa, plantea que la recuperación de los relatos de vida (life stories) e historias de vida (life histories) "constituyen una herramienta incomparable de acceso a lo vivido subjetivamente, y la riqueza de sus contenidos es una fuente de hipótesis inagotable" (p. 2). Ferraroti (2007), por su parte, plantea que las historias de vida, en particular, permiten estudiar al hombre no como un dato, sino como un proceso, es decir, permiten estudiar su historicidad. Arfuch (2002) sostiene que las ciencias sociales se han volcado cada vez más al testimonio y a la voz de los sujetos, con una intención de subrayar las experiencias colectivas pero desde trayectorias individuales.

En este marco, Robert L. Miller (2000), investigador de la Universidad Queen en Belfast (Irlanda del Norte), partiendo de los planteamientos de Bertaux (1999) sobre genealogías sociales comentadas y comparadas, plantea que en la investigación social contemporánea muchos investigadores buscan alejarse de la mirada atomista que produce la entrevista de una sola persona. En consecuencia, buscan nutrirse de otras fuentes para contar con un contexto más complejo sobre la vida de un individuo, a través de la entrevista a familias enteras, a generaciones diferentes de la misma familia. Tal como lo plantea el mismo Bertaux,

En el método de genealogías sociales comentadas y comparadas, la unidad de observación no es el individuo, sino un conjunto de trayectorias de vida de los individuos (y familias nucleares) conectados por relaciones de parentesco. La idea básica es definir la unidad de información así como incluir varias generaciones (al menos tres). (1995, p. 75).

Para Bertaux (1999), la GSCC parte de la recolección de genealogías sociales e historias de familias cuyo foco es la movilidad social. Esto quiere decir que este tipo de investigación se ocupa de recrear las trayectorias individuales de los descendientes a partir de intereses, deseos y contingencias relacionados con 
orientaciones, capitales y condiciones otorgados por sus figuras parentales y de autoridad. Bertaux (1999) señala que la investigación puede variar dependiendo del grado de ambición y de articulación-tensión con otras figuras que inciden en los descendientes, como la escuela, la iglesia, el partido o el mercado de trabajo.

En relación con la familia, el autor dice que a pesar de los múltiples intentos para declarar "la muerte de la familia" (p. 41), el interés por investigar lo que ocurre en este grupo -independientemente de su forma de configuración-, en términos de relaciones, soporte emocional, vínculos y demás, sigue estando en las primeras líneas de la investigación social.

En el trabajo que realiza Miller (2007) en el Centro de Investigación de Pobreza Crónica, asociado a la Escuela de Sociología de la universidad mencionada, sobre la transmisión intergeneracional de la pobreza, establece este vínculo entre las historias de vida individuales con las historias de familia. Entre otros asuntos, Miller (2007) señala que las historias de familia le permiten: poner los resultados cuantitativos y cualitativos en contexto; obtener una mirada holística de la familia; indagar la historia de la familia aún con miembros que viven separados de ella; y analizar los cambios generacionales en relación con la persistencia de la pobreza en diferentes generaciones de la misma familia.

Por otra parte, las historias de familia buscan problematizar la sociogénesis en torno a la relación sociedad-familias-individuos a partir de los procesos de figuración. Para Elias (2008), la figuración es un modelo cambiante que constituye a los jugadores (actores sociales) como totalidad. Estos no solo ponen en escena el intelecto sino toda su persona: su hacer, sus omisiones y sus relaciones mutuas. Las interdependencias no solo incluyen alianzas sino también oposiciones. En sus palabras,

[...] en el centro de las cambiantes figuraciones o del proceso de figuración hay un equilibrio fluctuante en la tensión, la oscilación de un balance de poder que se inclina unas veces más a un lado y otras más a otro. (p. 154).

De esta manera, la construcción del yo opera a partir de cadenas de interdependencias que sitúan al sujeto como individuo relacional en estructuras sociales, las cuales, a lo largo de la modernidad, fueron más o menos estables, pero que hoy se encuentran en proceso de redefinición.

Si bien las figuraciones en la modernidad se inscriben en el modelo civilizatorio que el propio Elias (2010) explica como mecanismo para incorporar la regulación, la vergüenza y la continencia en los grupos sociales, actualmente estas contienen nuevas características. El modelo de crianza, de socialización y de educación que proveen la familia y la escuela modernas, el cual resultó estable a lo largo de los últimos 250 años, se ha quebrado en la medida que las figuras sociales están perdiendo su capacidad de coerción. Incluso estas figuras, tradicionalmente ancladas en lenguajes, prácticas y relaciones jerárquicas, también se están desestabilizando en sus composiciones y dinámicas. Esto plantea nuevos desafíos de carácter teórico y metodológico para la investigación de las familias contemporáneas.

Por último, vale señalar que este método de investigación fue utilizado para abordar las experiencias de crianza en contextos de conflicto armado en Colombia. Las dinámicas que constituyen dichos contextos, en cierta medida, interpelan algunos aspectos planteados por Bertaux (1999) y Elias $(2008,2010)$ desde el mundo europeo. En consecuencia, más allá de explorar la deseabilidad de los progenitores o la movilidad que los descendientes logran gracias a los capitales y habilidades que heredan, la investigación en mención propuso la exploración de saberes, prácticas y valores de los integrantes de las familias para enfrentar las situaciones de violencia asociadas con el conflicto armado, para asumir el duelo por los familiares fallecidos a causa de la guerra y para construir motivaciones que les permitan seguir adelante en condición de sobrevivientes más que de víctimas.

\section{Criterios conceptuales y procedimentales para la realización de historias familiares}

Siguiendo la lógica de la investigación cualitativa, la elección de la muestra depende de los objetivos del 
estudio y de las características mismas de las personas que la componen, dado que darán una visión única a la investigación. En el caso de las historias de familia, habrá que tener en cuenta consideraciones adicionales, como la existencia de miembros de al menos tres generaciones de la familia a entrevistar, para cumplir con el objetivo de esta metodología, que implica situar en el contexto histórico-generacional el problema de investigación.

A partir de la elección de un "sujeto objetivo", se inicia la construcción de la genealogía social: "un mapa o diagrama de al menos tres generaciones de la familia que tomarán la forma de una red de relaciones de parentesco" (Miller, 2007, p. 8). Las condiciones que se espera tengan las tres generaciones son:
- Adultos jóvenes de la tercera generación (pueden ser niños o adolescentes siempre y cuando puedan dar una entrevista en profundidad sobre la historia de la familia).

- Adultos de mediana edad que sean lo suficientemente mayores para tener hijos jóvenes del primer grupo.

- Adultos mayores.

Es más fácil garantizar la presencia de las tres generaciones si se elige como "sujeto objetivo" (so en la gráfica) un miembro de los dos primeros grupos. La estructura básica de un mapa de historia de familia sería así como se muestra en la gráfica 1.

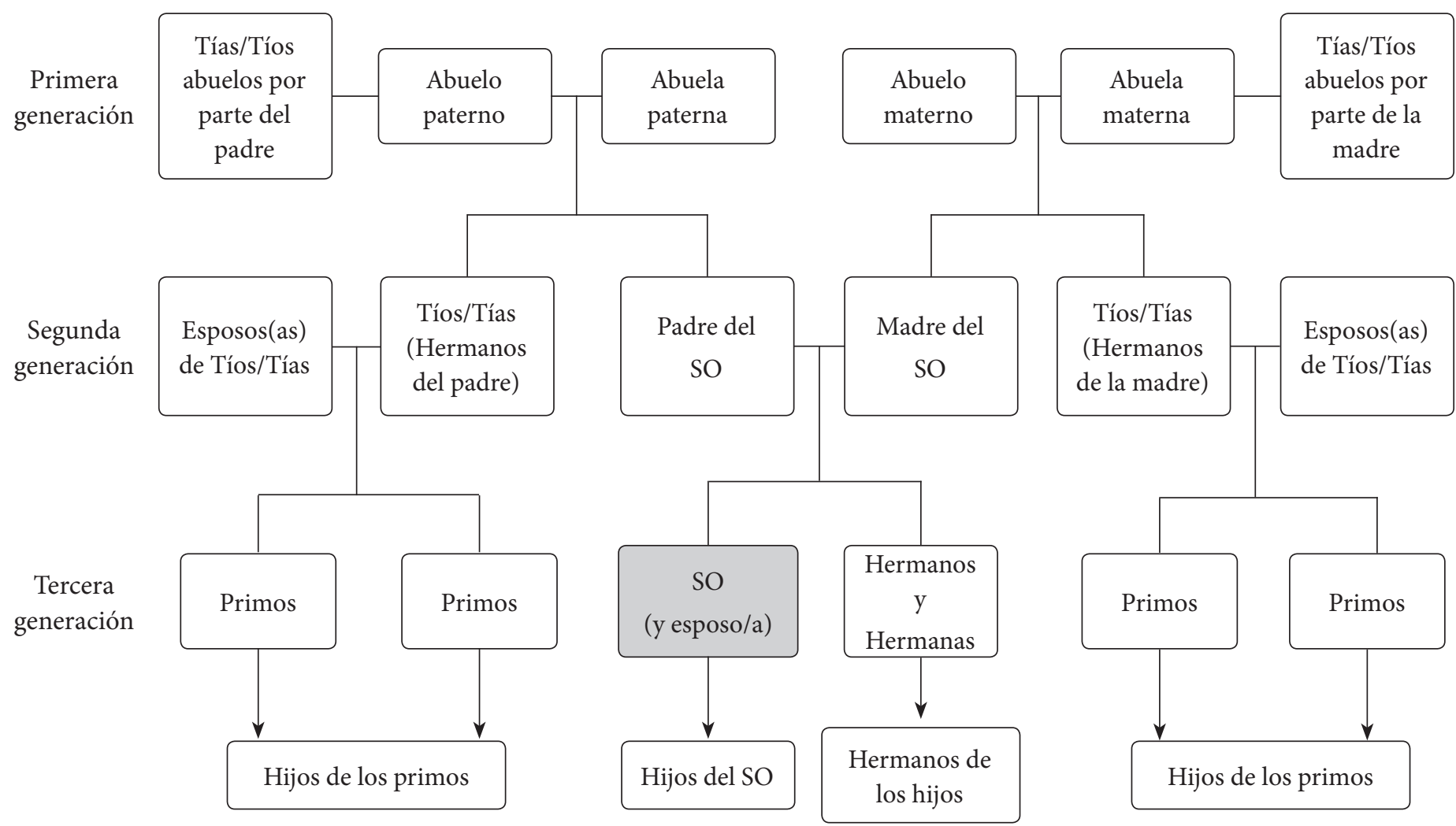

Gráfica 1. Estructura básica de un mapa de historia de familia

Fuente: Miller, 2007, p. 17, traducida del original. 
Los sujetos se ordenan de izquierda a derecha, ubicándose los mayores a la izquierda. Entre otros aspectos (y dependiente del objetivo de la investigación), se recomienda solicitar la siguiente información de cada miembro de la familia4:

- Nombres.

- Año de nacimiento/lugar de nacimiento.

- Años de escolarización/nivel instrucción más alto.

- Trabajo más común durante la vida.

- Ubicación actual (última ubicación), (si se murió).

- Otras notas.

Según Miller (2000), la construcción de los mapas de la historia familiar tiene dos propósitos: a) proporcionar un marco para las entrevistas de la historia familiar; y b) ser una fuente valiosa de información en sí mismo. Este mapa se construye con el "sujeto objetivo", y, una vez terminado, se seleccionan hasta cinco miembros de la familia (de las diferentes generaciones) para entrevistas adicionales.

La selección de esos cinco miembros mencionados en el párrafo anterior tiene que estar basada en el muestreo teórico, es decir, deben representar relevancia para la investigación, y deben cumplir con características de diversidad importantes para la saturación de la información (diferentes géneros, condiciones sociales, posiciones dentro de la familia, entre otros), que puedan captar la construcción de la historia familiar de la manera más completa $-\mathrm{y}$ compleja-posible.

4 Es importante anotar que el autor hace una aclaración en relación con estos ítems y es que ellos deben revisarse según el contexto cultural y social en el que se realiza la investigación, dado que puede no ser relevante alguno de los temas y quedar por fuera otros propios de la idiosincrasia del territorio donde son hechas las entrevistas.
La entrevista con dichos miembros sigue más o menos la misma estructura que la del sujeto objetivo, teniendo en cuenta que los desacuerdos o "contradicciones" encontradas en el discurso de los diferentes integrantes de la familia constituyen en sí mismos una fuente valiosa para el análisis de la historia familiar. Las migraciones de los miembros de la familia, por ejemplo, pueden ser una fuente importante de información en relación con los procesos de movilidad que han llevado a algunos integrantes de esa familia a tomar decisiones de marcharse del lugar de origen.

Siguiendo a Covarrubias (2004), la historia de familia se potencializa cuando los eventos narrados adquieren una dimensión temporal continua que relaciona el tiempo individual, el familiar y el histórico. En consecuencia, la historia de familia estimula la memoria social sobre acontecimientos desde abajo, e incluso pueden constituir otros marcos de comprensión del pasado. Tanto las perspectivas de la vida como las experiencias de pasado son narradas y resignificadas por los integrantes de las familias. En la mayoría de ocasiones, es necesario activar los recuerdos a través de objetos portadores de memoria y de otras narrativas, como las fotografías, las cartas, los textos, el mobiliario y la iconografía familiar puesta en escena en el lugar de vivienda. Es importante, según Cavarrubias (2004), explorar con técnicas precisas esas otras ecologías sociales.

En la gráfica 2 se presenta el mapa introductorio de una de las familias de la investigación. Los fondos blancos representan las personas que dieron la entrevista; la que está en letra roja es lo que se conoce como "sujeto objetivo" en las historias de familia (es decir, desde donde arranca la construcción de los mapas); y el resto, de fondo café, son los demás integrantes de la familia. Las cruces significan las personas que ya murieron. Los NN corresponden a personas que son de la familia (en términos de consanguinidad), pero no son identificados o reconocidos por el sujeto objetivo. 


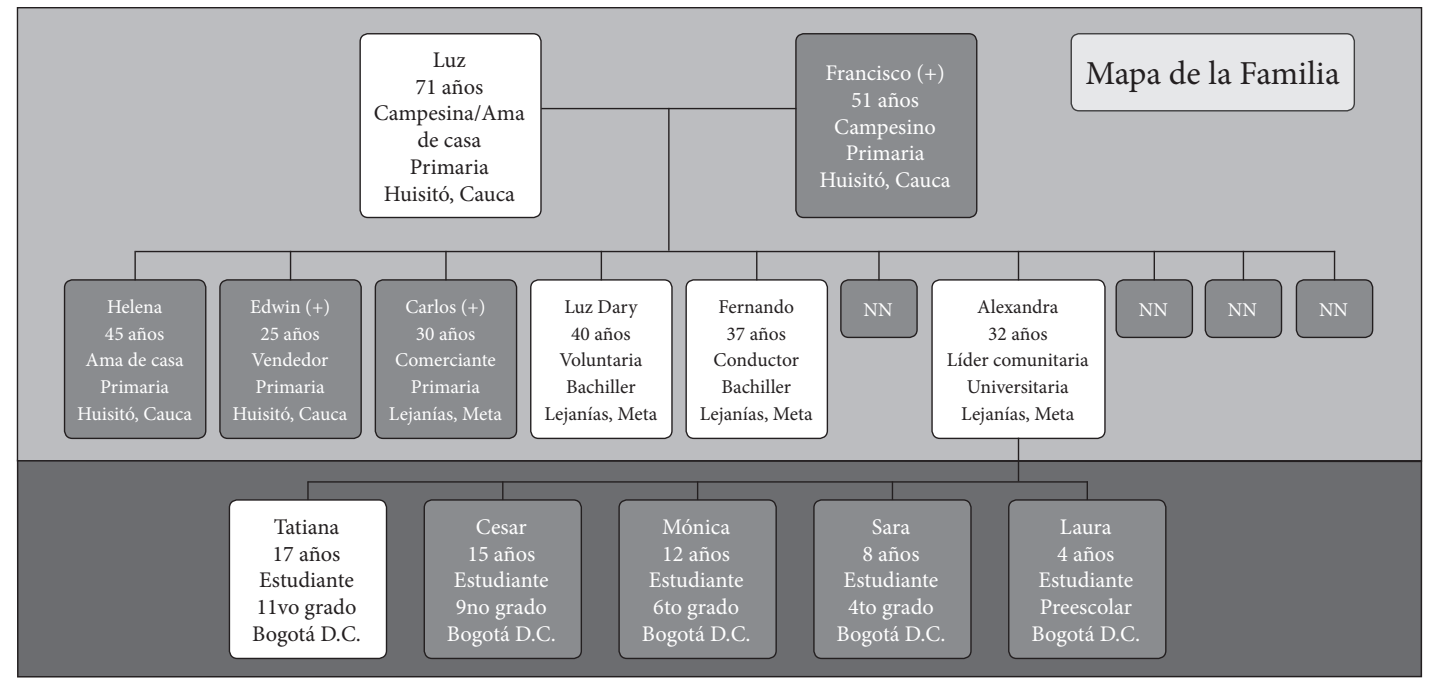

Gráfica 2. Mapa de familia

Si bien el mapa parece un genograma convencional, esta organización de la información, realizada de manera conjunta con los integrantes de la familia, a partir de la ubicación de las generaciones, las relaciones de parentesco, las edades, los tiempos, los momentos, las sucesiones y las secuencias, contribuye a que estos efectúen procesos de rememoración no como verdad de los hechos sino como recreación de lo pasado en el presente. Cavarrubias (2004) afirma que las personas de mayor edad se vuelven centrales para contar múltiples historias:

Generalmente tienen mayor información, disposición de tiempo y habilidad para narrar los acontecimientos. Muchas veces coincide que estas personas suelen ser mujeres: son las abuelitas, las señoras de la casa o la hija mayor. Pudiera suceder también que un informante clave con estas características sea varón, por ejemplo, el abuelo o un tío abuelo o el mismo padre de la familia. (p. 119).

Por otra parte, la historia de familia debe permitir la reconstrucción de la escala espacial que ha configurado la estructura familiar. En tal sentido, las entrevistas, los diálogos, los grupos focales y los talleres de memoria deben favorecer la reconstrucción de las espacialidades que han dado lugar a la sociogénesis entre lo societal, la familia y los individuos, conforme a lo expuesto por Elias (2010). En las gráficas 3 y 4 se presenta un esquema que representa la coordenada espacial que ha configurado a la familia ejemplificada.

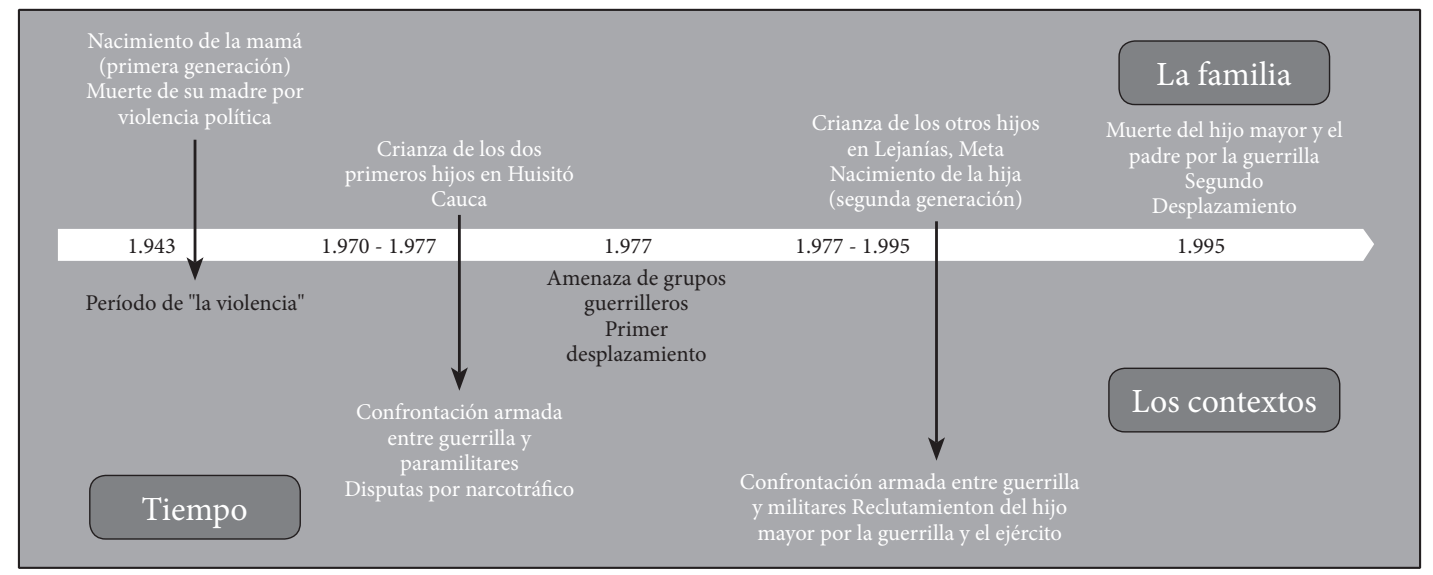

Gráfica 3. Coordenada espacial de una historia familiar 


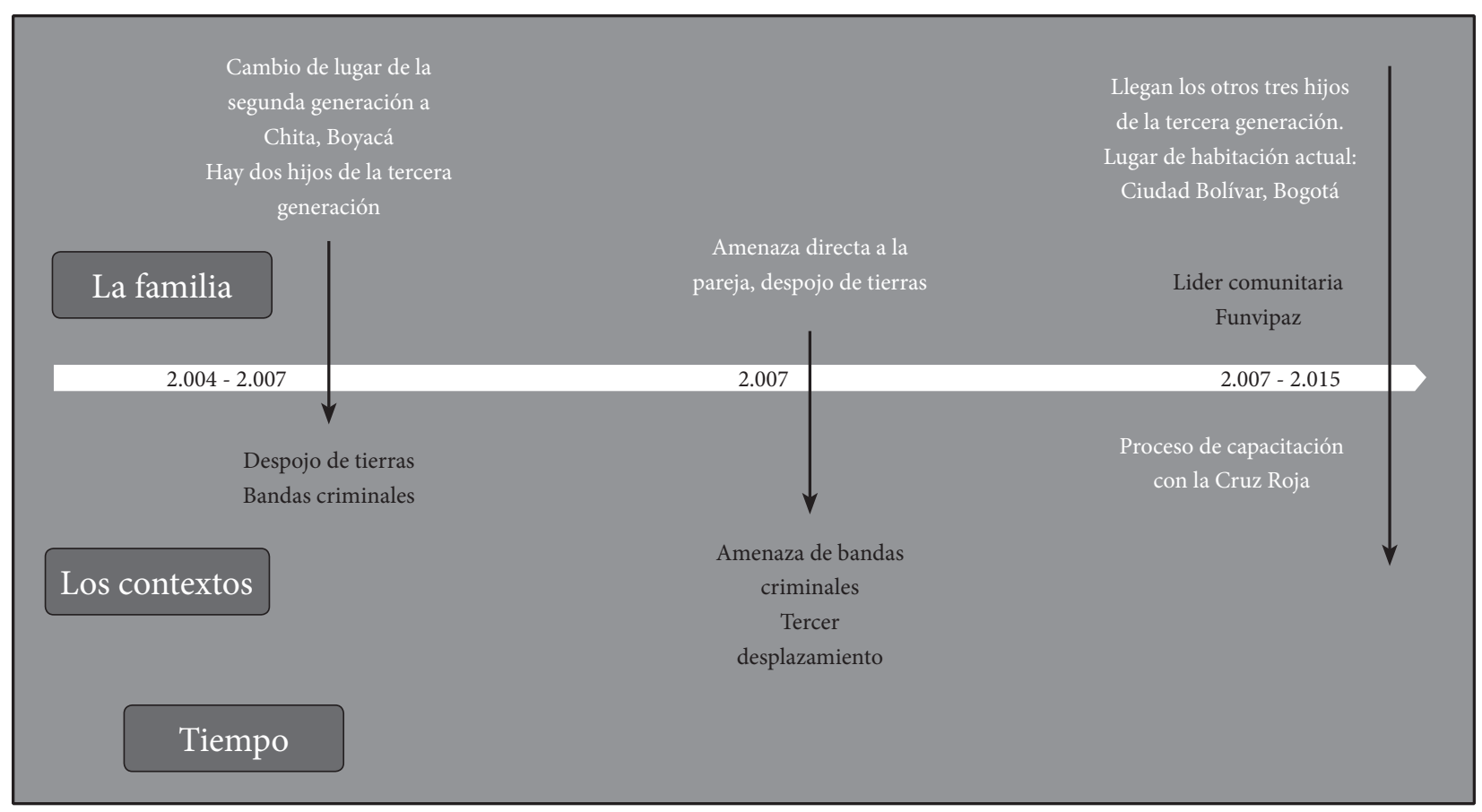

Gráfica 4. Coordenada espacial de una historia familiar

\section{Conclusiones}

Las historias de familia como método de recolección de datos se inscriben en la tradición biográfico-narrativa de las ciencias sociales, al tener en cuenta que estas abordan a las familias desde la producción de narrativas que se entretejen no solo con la historia particular del grupo familiar, sino, $y$ de manera significativa, con los contextos sociales, históricos y políticos en los que ella se encuentra inmersa. Así, entonces, las historias de familia proponen la creación de mapas familiares para señalar las relaciones que se establecen con los momentos y contextos históricos en que una familia vive.

Asimismo, esta metodología emplea herramientas interpretativas asociadas con la sociogénesis y las genealogías sociales abordadas por ciertas ramas de la sociología para comprender las permanencias y cambios de estas agrupaciones sociales, en un marco más amplio que el solo grupo familiar. En este sentido, es consecuente con los cambios que han impactado a la familia como institución social, dado que evidencia la complejidad de las relaciones más allá de los vínculos exclusivamente consanguíneos. En el levantamiento de los datos para hacer historias de familia con sus correspondientes mapas, se incluyen las personas que la familia considera como tales, sin restricciones de parentesco y consanguinidad. Así, la utilización de este método puede ser una contribución al proceso de deconstrucción de conceptos, discursos y textos sobre las familias contemporáneas.

En la vía de la construcción de memoria colectiva, las historias de familia permiten la expresión más compleja de los entrecruzamientos que se dan entre las historias de las personas y las historias de una sociedad. De esta manera, el contexto en el que se desarrollen tendrá un lugar relevante en la reconstrucción de la memoria histórica de ese contexto, porque es en ella donde se inscriben las producciones de las familias y los sujetos que las integran. De esta forma, en la investigación social hoy en día se hacen particularmente necesarias para acercarnos a las formas plurales y particulares de narrar la propia historia.

Finalmente, hay que volver a señalar que las historias de familia como metodología no son genogramas ni hacen parte del estudio de las tipologías familiares en función de su estructura. Es importante resaltar que estas se inscriben en una tradición que no pretende diagnosticar "los problemas familiares" 
sino señalar las trayectorias de vida de las familias y los sujetos que las integran, en el marco ampliado del momento histórico en el que dichos grupos se conformaron.

\section{Referencias}

Arfuch, L. (2002). El espacio biográfico. Dilemas de la subjetividad contemporánea. Buenos Aires: Fondo de Cultura Económica.

Berger, P. y Luckman, T. (2005). La construcción social de la realidad. Madrid: Amorrortu.

Bertaux, D. (1999). El enfoque biográfico: su validez metodológica, sus potencialidades. Proposiciones, 29, 1-22.

Burin, M. y Meler, I. (2003). Género y familia: Poder, amor y sexualidad en la construcción de la subjetividad. Buenos Aires: Paidós.

Covarrubias, K. (2004). Historias de familia: Una propuesta metodológica para el estudio de la pobreza en familias evangélicas de Colima, México. Estudios sobre las Culturas Contemporáneas, X(20), 107-139.

Coffey, A. y Atkinson, P. (2003). Encontrar sentido a los datos cualitativos. Estrategias complementarias de investigación. Medellín: Contus, Universidad de Antioquia.

Creswell, J. (1998). Qualitative inquire and research design. Choosing among five tradictions. California, Thousand Oaks: Sage.

Elias, N. (2008). Sociología fundamental. Barcelona: Biblioteca Económica Gedisa.
Elias, N. (2010). El proceso de la civilización. México: Fondo de Cultura Económica.

Ferraroti, F. (1988). Biografía y ciencias sociales. San José de Costa Rica: Flacso.

Habermas, J. (2007). Conocimiento e interés. En Conformismo o democracia. Bogotá: Fica.

Ibañez, J. (1990). Nuevos avances en la investigación social. La investigación social de segundo orden. Barcelona: Anthropos.

Kaufman, F. (2005). Metodología de las ciencias sociales, E. Imaz (trad.). México: Editora Nacional.

Lakoff, G. y Johnson, M. (1980). Metáforas en la vida cotidiana. Chicago: Universidad de Chicago.

Miller, R. L. (2000). Researching life histories and family histories. Londres: Sage.

Miller, R. L. (2007). Using family histories to understand the Intergenerational Transmission of Chronic Poverty. CPRC Working Paper 103. Belfast: Queen's Univertisty.

Morse, J. (2003). Asuntos críticos en los métodos de investigación cualitativa. Medellín: Contus, Universidad de Antioquia.

Santos, B. (2009). Una epistemología del sur. México: Siglo xxi/Clacso.

Spivack, G. (2003). ¿Puede hablar el subalterno? Revista Colombiana de Antropología, (39), 297-364.

Vasilachis, I. (2006). Estrategias de investigación cualitativa. Buenos Aires: Paidós.

Walsh, C. (Ed.) (2012). Pedagogías decoloniales. Prácticas insurgentes de resistir, (re)existir y (re)vivir. Tomo I. Quito: Universidad Andina Simón Bolívar. 